\title{
H Pylori Infection as Risk Factor for GIT Bleeding in Haemophilic Patients
}

\author{
Noha M El Husseiny*, Louis Essac and Mona Al Kassas \\ Clinical Haematology Unit, Internal Medicine Department, Kasr Al Aini, Faculty of Medicine, Cairo University, Egypt \\ ${ }^{*}$ Correspondence to: Noha M El Husseiny, Clinical Haematology Unit, Internal Medicine Department, Kasr Al Aini, Faculty of Medicine, Cairo University, Egypt; \\ E-mail: noha_el_husseiny77@yahoo.com
}

Received: November 21, 2016; Accepted: December 07, 2016; Published: December 14, 2016;

\begin{abstract}
Background: Helicobacter pyelori is endemic in Egypt and present a main cause of gastrointestinal bleeding.
\end{abstract}

Aim: Aim of this study is to evaluate the prevalence of Hpylori infection in hemophilic patients, and to assess its impact on gastrointestinal bleeding associated with this infection in such patients.

Methods: we prospectively investigated the prevalence of H. pylori infection in 40 Egyptian patients with hemophilia A, B and von Willebrand syndrome and 20 normal male subjects were included. Every patient and control subject in the study were tested one time for H. pylori stool antigen by ELISA. All patients and control subjects were tested for occult blood using Guaiac-based fecal occult blood test.

Results: Twenty eight out of 40 patients (70\%) are H. pylori positive ; and 12 out of 20 control (60\%) are H. pylori positive. The odds ratio is 1.55 , $95 \%$ CI (0.6162 to 3.9269), Significance level P = 0.3497. Among 28 H.pylori positive patients, 5 patients $(17.9 \%)$ tested positive for occult blood. Among the $12 \mathrm{H}$.pylori positive subjects in the control group, only one tested positive for occult blood (8.3\%). Odds ratio for Occult bleeding in $\mathrm{H}$ pylori positive patients and control was 2.39: $\mathrm{P}=0.4504$. None of the $\mathrm{H}$. pylori negative patients or control subjects had a positive occult blood disease.

Conclusion: patients with hemophilia, H. pylori should not be considered as an important cause of GI bleeding. The recurrence of the infection and GI bleeding could be prevented with eradication of H. pylori. Screening tests for H. pylori would not be needed in patients with hemophilia in endemic areas.

Key words: Haemophilia, Hpyelori

\section{Introduction}

In Egypt, it is estimated that 5050 people with haemophilia and, 499 with vWD are registered within the six hemophilia treatment centers (www.wfh.org annual report 2013)

More than $50 \%$ of the world's population harbor Helicobacter pylori in their upper gastrointestinal tract. Infection is more prevalent in developing countries, and incidence is decreasing in Western countries. [1]

According to world gastroenterology organization report in 2010, H.pylori incidence in adult Egyptians was 90\% (www. worldgastroenterology.org)

It has been hypothesized that the host immunologic response against $\mathrm{H}$. pylori plays a main role in determining gastric mucosal injury, through the release of cytokines and the action of autoantibodies against $\mathrm{H1} / \mathrm{K} 1$-adenosine triphosphatase of gastric epithelial cells. [2]

Infection with $\mathrm{H}$ pylori is the main etiological factor for erosive gastritis and duodenal or gastric peptic ulcers that often complicat with life-threatening bleeding in patients with coagulation disorders. [3]

The Egyptian government provides approximately 38\% of healthcare costs of treating haemophilic patients (www.wfh.org annual report 2013). Most hemophilia patients are treated with plasma or cryoprecipitate, resulting in a high risk of transfusing blood-borne diseases like HIV and hepatitis B and C. Factor concentrate is available only in limited quantities, and patients must travel long distances to get it.

There is a contradictory in the previous studies between prevalence of $\mathrm{H}$ pyelori and risk of GIT of bleeding, we prospectively investigated the prevalence of $\mathrm{H}$. pylori infection in patients with hemophilia $\mathrm{A}$ or $\mathrm{B}$ or von Willebrand syndrome and it is impact on risk of bleeding in those patients and compare our results with others'.

\section{Patients and methods}

This is a case control study. All participants are adult patients visiting the hematology clinic of kasr Al Aini hospital and the Egyptian society of Hemophilia. The study received research ethical committee approval from Kasr Al Aini medical school and all participants gave written consents. Forty patients with bleeding disorders (mean age 24); categorized as: 30 patients with Hemophilia A, 6 patients with Hemophilia B and 4 patients with VWD. Patients who received triple therapy before or Patients with liver cirrhosis, thrombocytopenia, any platelet or vascular defects or patients with peptic ulcer were excluded. Control group included 20 subjects (mean age 27) of the 
same socioeconomic level and the same exclusion criteria of the patients included in the study. Each Patient and control subject in the study were tested for $\mathrm{H}$. pylori stool antigen by ELISA, This technique is non-invasive, rapid, easy-to-use, and shows good performance characteristics for diagnosis of $\mathrm{H}$. pylori infections, with sensitivity, specificity, Positive predictive value (PPV), negative predictive value (NPV), and efficiency were $100 \%, 90.0 \%, 96.9 \%, 100 \%$, and $97.6 \%$ respectively).[4] All Patients and control subjects were tested for occult blood using guaiac-based fecal occult blood test. [5] All diet and drug precautions were applied before obtaining samples to prevent false positive results as dietary restriction of both hemoglobin and vegetable peroxidase containing substances is essential for valid screening for occult blood.[6]

\section{Statistics}

Using SPSS V.16.0 for calculation of the means and $\mathrm{z}$ test for comparison between the prevalence of $\mathrm{H}$. pylori infection among Hemophilia A, Hemophilia B and von Willibrand patients and the prevalence of $\mathrm{H}$. pylori infection among the control group to investigate whether there is a difference in prevalence between both groups or not.

Comparison between the odds ratio (OR) of developing occult GI bleeding (indicted by positive guaiac test) among Hemophilia A, Hemophilia B and von Willibrand patients who test positive for $\mathrm{H}$. pylori and the odds ratio of developing occult GI bleeding among the control group who test positive for $\mathrm{H}$. pylori to investigate whether there is a relation between the possible risk and the outcome.

\section{Results}

The patients' group included 40 male patients (30 patients with Hemophilia A (75\%), 6 patients with Hemophilia B (15\%), and 4 patients with VWD (10\%)) with age ranged from 12 to 52 years (mean age 24 years).

Twenty eight out of 40 patients (70\%) are H. pylori positive; and 12 out of 20 controls (60\%) are H. pylori positive. Odds ratio: 1.55) 95\% Confidence Interval: 0.6162 to 3.9269; $\mathrm{P}=0.3497$ )

Among $28 \mathrm{H}$. pylori positive patients, 5 patients tested positive for occult blood (17.9\%). all patients positive for occult blood were also positive for $\mathrm{H}$. pylori stool antigen. Among the 12 subjects positive for $\mathrm{H}$. pylori in the control group subjects, only one tested positive for occult blood ( $8.3 \%$ ), and he was also $\mathrm{H}$. pylori positive. Odds ratio was 2.3913 (95\% Confidence Interval: 0.2485 to 23.0104; $\mathrm{P}=0.4504$ ). None of the $\mathrm{H}$. pylori negative patients or control subjects had a positive occult blood disease.

Table 1 and Figure 1 demonstrate the prevalence of H.pylori and occult bleeding in patients and control.

\section{Discussion}

The prevalence of $\mathrm{H}$. pylori infection in patients with hemophilia A or B or von Willebrand syndrome was investigated and compared to the prevalence of $\mathrm{H}$. pylori infection in normal control subjects in many studies with variable results. In our study, we didn't find significant difference between prevalence of $\mathrm{H}$. pylori among hemophilia and VWD patients $(70 \%) \mathrm{n}=40$ and its prevalence among normal control subjects $(60 \%) \mathrm{n}=20$, the odds ratio is $1.55,95 \% \mathrm{CI}$ (0.6162 to 3.9269), z statistic 0.935 , Significance level $\mathrm{P}=0.3497$ ). The Prevalence of $\mathrm{H}$. pylori revealed by this study doesn't differ from the reported prevalence in general population: 72.38\% .[7] While the odd ratio of $\mathrm{H}$. pylori infection is about 1.5; It is insignificantly higher for occult bleeding detected by FOBT in absence of history of frank bleeding; Odd ratio is $2.39,95 \%$ Confidence Interval: 0.2485 to $23.0104,, \mathrm{P}=0.4504$. Our case and control were matched for geographic distribution, age, socio economic status and educational level to avoid misleading results as described previously by wang. [8] Our results are also concomitant with Braden et al who found similar prevalence of Helicobacter pylori Infection in seventy patients with haemophilia and WVD and 100 age-related volunteers $P$ value were statistically insignificant.[9] Also Eleftheriadis studied thirty-seven patients with hereditary haemorrahgic diseases and 26 control. ELISA was used to detect IgG, anti-CagA, and IgA antibodies to H. pylori in the serum and saliva. Result of this study revealed that $64.8 \%$ of the patients and $65.4 \%$ of the controls had $\mathrm{H}$. pylori IgG antibodies in serum $(\mathrm{P}=0.1$ (. [10] Szczepanik also found that the prevalence of $\mathrm{H}$. pylori infection in hemophilic patients in Poland was comparable to that in patients without coagulation disorders (49.3\% vs $39 \%$ respectively $\mathrm{P}=0.11$ ). [3]

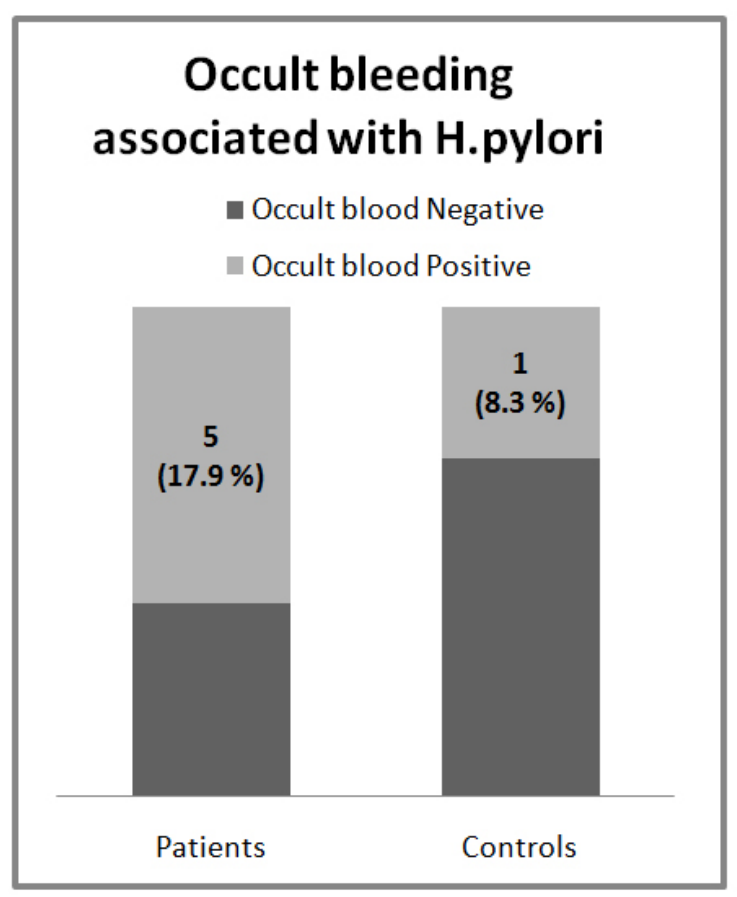

Figure.1 Prevalence of occult bleeding in patients and control

However our results were inconsistent with the study of upper gastrointestinal bleedings in patients with hereditary coagulation disorders in Northwest of Iran. The prevalence of H. pylori infection was investigated by stool antigen test, and serum serologic tests including immunoglobulin G and anti-CagA. Results Among 90 patients (81 men, nine women, mean age $31.30 \pm 10.72$ years), 66 patients with hemophilia A, 10 patients with hemophilia B, six patients with Von 
Willebrand disease, five patients with platelet function disorders, and three patients with other factor deficiencies were evaluated. About $46.7 \%$ of patients in group A, and $23.3 \%$ of patients in group B were anti-CagA-positive $(\mathrm{P}=0.02)$, whereas $76.7 \%$ of patients in group $\mathrm{A}$ and $51.7 \%$ of patients in group B had H. pylori immunoglobulin G antibodies $(\mathrm{P}=0.02)$. $\mathrm{H}$. pylori antigen in stool was positive in $76.7 \%$ in group $\mathrm{A}$ and $55 \%$ in group $\mathrm{B}(\mathrm{P}=0.03)$. [11]

Table 1. Prevalence of H.pylori and occult bleeding in patients and control

\begin{tabular}{|l|c|c|c|c|}
\hline \multicolumn{1}{|c|}{ Diagnosis } & \multicolumn{2}{|c|}{ Occult Blood Positive } & \multicolumn{2}{c|}{ Occult blood negative } \\
\hline & $\begin{array}{c}\text { H. pylori } \\
\text { Positive }\end{array}$ & $\begin{array}{c}\text { H. pylori } \\
\text { Negative }\end{array}$ & $\begin{array}{c}\text { H. pylori } \\
\text { Positive }\end{array}$ & $\begin{array}{c}\text { H. pylori } \\
\text { Negative }\end{array}$ \\
\hline Hemophilia A & 5 & 0 & 15 & 10 \\
\hline Hemophilia B & 0 & 0 & 4 & 2 \\
\hline VWD & 0 & 0 & 4 & 0 \\
\hline Total & 5 & 0 & 23 & 12 \\
\hline
\end{tabular}

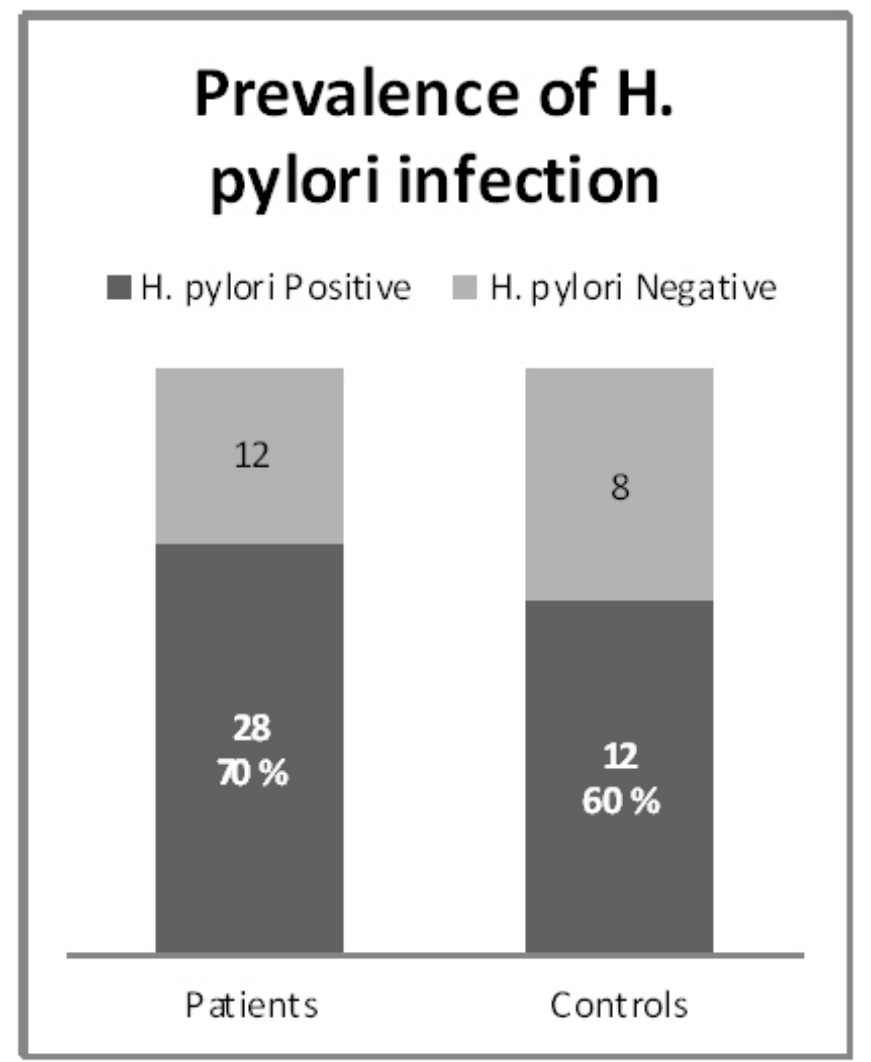

Figure.2 Prevalence of H.pylori in patients and control

Our study also found statistically insignificant increased risk of fecal occult bleeding in absence of history of frank bleeding in patients with hemophilia A; Odds ratio was 2.39, 95\% (Confidence Interval: 0.2485 to 23.0104, $z$ statistic: 0.755 , Significance level: $\mathrm{P}=0.4504$ ).

Though the prevalence of H pylori in Szczepanik et al. study were statistically insignificant however the gastrointestinal bleeding was reported in 46 patients $(31.5 \%)$ with hemophilia and in two control group patients $(2.0 \%)(\mathrm{P}<0.0001)$. Gastrointestinal bleeding was significantly more frequent in patients with hemophilia infected with
H. pylori $(33 / 46 ; 71.7 \%)$ than in patients with no $\mathrm{H}$. pylori infection (13/46; 28.3\%; P $=0.0002)$. Concluding that Helicobacter pylori infection is a risk factor for duodenal and gastric ulcer bleeding in hemophilia patients. [3]

Eyster et al detected antibodies against H. pylori in 14 (35\%) of 40 subjects who had upper gastrointestinal bleeding event compared with 7 (17\%) of 41 controls who were closely matched for age and other factors. Bleeding was substantially but not significantly increased (OR: 4.6; 95\% CI: 0.3-83.9) with H. pylori seropositivity. [12]

Though Choe studied a group of haemophilic children but his results concluded significant role of $\mathrm{H}$ pylori in inducing GIT bleeding. Helicobacter pylori infection was found in four of six (66.7\%) patients with GI bleeding (3, duodenal ulcer; 1, H. pylori associated chronic gastritis). The patients with $\mathrm{H}$. pylori infection had an eradication treatment of triple therapy and no recurrence happened. [13]

According to our results screening for $\mathrm{H}$. pylori should be routine work-up for all patients with hemophilia $\mathrm{A}$, and all patients with positive stool antigen test should be treated immediately to prevent gastrointestinal bleeding, and to repeat testing to ensure eradication of the bacteria which may definitely lower the costs as was described by Schulman.[14]

\section{Conclusion}

Patients with hemophilia, H. pylori should not be considered as an important cause of GI bleeding. The recurrence of the infection and GI bleeding could be prevented with eradication of $H$. pylori. Screening tests for $\mathrm{H}$. pylori would not be needed in patients with hemophilia in endemic areas.

\section{Ethical approval}

All procedures performed in studies involving human participants were in accordance with the ethical standards of the institutional and/ or national research committee and with the 1964 Helsinki declaration and its later amendments or comparable ethical standards.

Informed consent was obtained from all individual participants included in the study.

Authors declare no conflict of interest. Authors declare that the results have not been published previously and are not under submission elsewhere.

\section{References}

1. Brown LM, (2000) Helicobacter pylori: epidemiology and routes of transmission. Epidemiol Rev 22: 283-297. [crossref]

2. Emilia G, Longo G, Luppi M, Gandini G, Morselli M, et al. (2001) Helicobacter pylori eradication can induce platelet recovery in idiopathic thrombocytopenic purpura. Blood 97: 812-4.

3. Szczepanik AB, Zaleska M, Wiszniewski A, Wislawski S, Misiak A, et al. (2005) Helicobacter pylori infection in patients with haemophilia in Poland: prevalence and risk of upper gastrointestinal bleeding. Haemophilia 11:376-9

4. Kim PS, Lee JW, Pai SH, Kim YB, Cho JK, et al. (2002) Detection of Helicobacter pylori antigen in stool by enzyme immunoassay. Yonsei Med J 43: 7-13. [crossref]

5. Green BT, Rockey DC (2004) Gastrointestinal endoscopic evaluation of premenopausal women with iron deficiency anemia. J Clin Gastroenterol 38:104-9

6. Syed AA, Khatoon BA, Silwadi MF (2001) New reagents for detection of faecal occult blood. J Pharm Biomed Anal 24: 581-586. [crossref]

7. Mohammad MA, Hussein L, Coward A, Jackson SJ (2007) Prevalence of Helicobacter pylori infection among Egyptian children: impact of social background and effect on growth. Public Health Nutrition 11: 230-236. 
8. Wang F, Meng W, Wang B, Qiao L (2014) Helicobacter pylori-induced gastric inflammation and gastric cancer. Cancer Lett 345: 196-202. [crossref]

9. Braden B, Wenke A, Karich HJ, Dietrich CF, Scharrer I, et al. (1998) Risk of gastrointestinal bleeding associated with Helicobacter pylori infection in patients with hemophilia or von Willebrand's syndrome. Helicobacter 3: 184-187. [crossref]

10. Eleftheriadis N, Makri S, Aggouridaki C, Pithara E, Makris P (2002) Helicobacter pylori infection in upper gastrointestinal bleeding in patients with hereditary hemorrhagic disorders. Eur J Intern Med 13: 480-4.

11. Dolatkhah R, Khoshbaten M, Asvadi Kermani I, Reza Bonyadi M, Ghojazadeh M, et al. (2011) Upper gastrointestinal bleedings in patients with hereditary coagulation disorders in Northwest of Iran: prevalence of Helicobacter pylori infection. Eur $J$ Gastroenterol Hepatol 23: 1172-7.
12. Eyster ME, Asaad SM, Gold BD, Cohn SE, Goedert JJ (2007) Upper gastrointestinal bleeding in haemophiliacs: incidence and relation to use of non-steroidal antiinflammatory drugs. Haemophilia 13:279-86.

13. Choe BH, Kim JY, Lee JH, Kim JM, Chu MA, Cho SM (2010) Upper gastrointestinal bleeding in children with haemophilia: a clinical significance of Helicobacter pylori infection. Haemophilia 16: 277-80

14. Schulman S, Rehnberg AS, Hein M, Hegedus O, Lindmarker P, Hellström PM (2003) Helicobacter pylori causes gastrointestinal hemorrhage in patients with congenital bleeding disorders. Thromb Haemost 89: 741-6.

\section{Citation:}

Noha M El Husseiny, Louis Essac and Mona Al Kassas (2016) H Pylori Infection as Risk Factor for GIT Bleeding in Haemophilic Patients. Internal Med Res Open J Volume 1(2): 1-4 